\title{
LOW GRADE ADENOMYOEPITHELIAL CARCINOMA OF THE BREAST - A CASE REPORT
}

Vaibhav Mane, S. R. Agashe, R. T. Joshi
1. Assistant Professor, Department of Pathology, Bharati Vidyapeeth Medical College and Hospital. Sangli, Maharashtra.
2. Professor, Department of Pathology, Bharati Vidyapeeth Medical College and Hospital. Sangli. Maharashtra.
3. Professor, Department of Pathology, Bharati Vidyapeeth Medical College and Hospital. Sangli. Maharashtra.

\section{CORRESPONDING AUTHOR}

Dr. Vaibhav P. Mane

Vaishnavi Pathology Laboratory, Shop No.2, Kore House, Vishrambag, Sangli

416415, Maharashtra,

E-mail: vaishnavilab@rediffmail.com

Ph: 00919422041490.

\begin{abstract}
Adenomyoepithelioma of breast is a rare benign neoplasm characterized by biphasic proliferation of epithelial and myoepithelial cells. (1,2,3,4,10).It occurs in older women and presents as unilateral painless ,palpable mass.(1,5,6,10 )Mammographic findings are nonspecific. (2).The tumour has a potential for local recurrence $(1,3)$ therefore wide excision is recommended. Histologically these tumours are characterized by epithelial cells in glandular pattern, while myoepithelial cells are spindle to oval cells with clear cytoplasm in sheets. Immunohistochemistry shows positivity of myoepithelial cells for SMA, S100, p63, desmin while epithelial cells show antibodies to cytokeratins. (1,2).Malignant transformation can occur rarelyc $2,3,5)$
\end{abstract}

KEYWORDS: Breast, adenomyoepithelioma, Low Grade Adenomyoepithelial carcinoma

INTRODUCTION: Adenomyoepithelioma of breast are uncommon tumours characterized by biphasic proliferation of epithelial and myoepithelial cells.(1,2,3) . It was first described by Hampel in 1970 and further classified by Tavassoli in 1991(3). It usually occurs in older patients, unilateral, painless breast lump being the commonest presentation.(5) All reported cases were female except for two male patients.(1)Although these are benign tumours, local recurrence is reported, therefore wide local excision is the treatment of choice.Rarely malignant transformation can occur in one or both the components (6).Differential diagnosis of this tumour includes sclerosing adenosis, fibroadenoma and tubular adenoma ( 1)

CASE REPORT: A 80-year-old female patient was admitted with complaints of gradually increasing painless mass in the left breast since last 4 years. Mammography of left breast showed large uniform lump measuring $8 \times 8 \times 7.1 \mathrm{~cm}$ occupying most of the breast. It has well defined margins, no satellite lesions, no speculations, no calcification in the lump. Axillary tail region was normal. Mammography of the right breast was normal.

Local examination revealed firm lump in left breast .The nipple and overlying skin was normal. No axillary lymphadenopathy was noticed.

Routine laboratory test were within normal ranges.

FNAC of the lump was done and was reported as benign breast lesion suggestive of phyllodes tumour. It is true the lesion of Phyllodes tumour is seen in younger group ,but on FNAC of this lesion the smears are cellular with both epithelial and more benign stromal 


\section{CASE REPORT}

fragments , atypia is absent. The possibility of Phyllodes is considered and biopsy is advised for confirmation.

THE PATIENT UNDERWENT LUMPECTOMY: Gross examination showed left simple mastectomy specimen with a gray white mass measuring $9.5 \times 5 \times 4 \mathrm{~cm}$. Cut section showed gray white appearance with cystic areas.

Microscopically the tumour showed biphasic nature composed of epithelial and myoepithelial cells. The epithelial cells are cuboidal to columnar with eosinophilic cytoplasm arranged in tubular pattern while the myoepithelial cells are round to oval with moderate amount of clear cytoplasm arranged in sheets. Mitotic activity is inconspicuous. In areas infiltrative pattern, foci of squamous metaplasia and areas of necrosis are noted. The nipple and areola are unremarkable .

In Differential diagnosis in this case the possibilities of tubular adenoma, sclerosing adenosis and fibroadenoma are ruled out because all these lesions usually occur in younger age group. Again the typical biphasic appearance of epithelial and myoepithelial component which is observed in this case is usually not seen in other lesion. The microscopic features seen in tubular adenoma ( closely packed uniform small tubules etc ) and in sclerosing adenosis ( Nodule with lobular configuration with more cellular centrally than peripherally ) is not noted in this case.

Immunohistochemistry showed the positivity for SMA, S100 and p63in the myoepithelial cells. The mitotic rate was around $6 / 10 \mathrm{hpf}$. So the final diagnosis was low grade adenomyoepithelial carcinoma.

DISCUSSION: Myoepithelial cells are normal component of breast tissue.( 1,6$)$. However myoepithelial neoplasms are uncommon in breast.(1,3,6,9).Tavassoli classified them into three categories myoepitheliosis, adenomyoepithelioma and myoepithelial carcinoma. $(1,3)$.

Adenomyoepitheilomas show biphasic proliferation of inner epithelial cells and peripheral layer of myoepithelial cells (1).The morphological appearance depends upon the relative amount of epithelial and myoepithelial component.

Adenomyoepitheilomas have been further divided into tubular, spindled or lobulated subtype depending upon their growth pattern $(1,3,9$,).

Tubular pattern is the commonest pattern (1) showing epithelial cells in tubular or glandular arrangement surrounded by myoepithelial cells with clear cytoplasm. Malignant changes in one or both components have been described (1)

The exact etiology of breast adenomyoepithelioma is not known. All cases are sporadic and no positive family history is noted (1) Kiaer et al described a case with sequential change from adenomyoepithelial adenosis to adenomyoepithelioma which eventually became low grade malignant

adenomyoepithelioma during a course of 18 years.(1,3,6)From these findings Choi et al proposed that adenomyoepitheliomas were derived from long standing myoepithelial breast lesions, such as adenosis and fibroadenoma $(1,3)$

Immunohistochemical confirmation of histologic diagnosis is always necessary. Immunohistochemistry shows that myoepithelial cells are positive for SMA, S100, p63 and desmin while epithelial cells show antibodies to cytokeratins. $(1,2)$

Diiferential diagnosis includes tubular adenoma, sclerosing adenosis and fibroadenoma $(1,3)$ Tubular adenoma, sclerosing adenosis and fibroadenoma show less proliferative features. 
Immunohistochemistry for myoepithelial component is must.

The prognosis of these patients is usually good. Wide local excision with adequate margins is the treatment of choice.(6)

Usually these lesions are benign but rarely they can become malignant.

High mitotic rate, cytologic atypia and infiltrative peripheral borders favour malignancy.(5)

Malignant myoepithelial tumours are either pure myoepithelial carcinoma or an adenomyoepithelioma with a component of myoepithelial carcinoma, epithelial carcinoma, sarcoma or carcinosarcoma, (6) Distant metastasis is very rare, in low grade tumours.

CONCLUSION: Adenomyoepithelioma of breast are rare tumours occurring in older female patients, presenting with unilateral mass .Radiological investigations are non contributory. Immunohistochemistry confirmation of histological diagnosis is necessary. Wide local excision with adequate margins is the treatment of choice.

\section{REFERENCES:}

1. Pei -Yi Chu, Tsung - Han Teng,TSui - Fen Cheng, Chin - Cheng Lee, Adenomyoepithelioma of the breast - A Case report, Department of Pathology and laboratory medicine, General Surgey, Shin Kong $\mathrm{Wu}$ Ho Su Memorial Hospital, Taipei,Taiwan Tzu Chi Med J 2006 . 18: 65 -67

2. Maura O'Neil, Fang Fan ,Iavn Damjanov.Department of Pathology and laboratory medicine ,The university of Kansas Medical Center,Kansas City,KS

3. Jin Sub Choi, Jong Yup Bae, Woo Hee Jung, Adenomyoepithelioma of the Breast - Its diagnostic problems and histogenesis. Yonsei Medical Journal Vol.37 No.4 1996

4. FAusta Catena, Donatella Santini et al .Adenomyoepithelioma of the Breast : An Intricate Diagnostic Problem .Breast Care 2008; 3 : 125 -127( DOI : 10.1159/000119727)

5. Hind Nassar: Breast - nonmalignant, Benign( usually ) tumors/tumor like changes, Adenomyoepithelioma. Pathology Outlines.com

6. Khurana Anil, Jalpota Yashpal. MYoepithelial carcinoma arising in an adenomyoepithelioma of the breast : A case report of a rare entity.Indian journal of Pathology and Microbiology 2010,Volume 53, Issur 2, Page 310 -312.

7. Plaza JA, Lopez JI, Garcia S, de Miguel C. Adenomyoepithelioma of the breast .Report of two casesArch anat Cytol PAthol $1993 ; 41$ (2): $99-101$

8. Hikino H, Kodama K, etal Intracystic adenomyoepithelioma of the breast - case report and review.Breast Cancer.2007; 14 ( 4): 429 -433

9. D.C.Howlett, C.H.Mason, S.BIswas.P.D.Sangle, G.Rubin, S.M.Allan. Adenomyoepithelioma of the Breast: Spectrum of Disease with Associated Imaging And Pathology. American Journal of Roentgenology.www.ajronline.org, AJR March 2003,Vol.180,No.3 : 799 -803

10. Asim Qureshi , Naila Kayani, Rubina Gulzar. Malignant adenomyoepithelioma of the breast: a case report with review of literature. BMJ Case reports2009, bcr01.2009.1442, Published online 2009 June 18.

11. Jatin Gandhi, Anurag Mehta et al . Malignant adenomyoepithelioma : A tumour of low malignant potential despite basal pheno . Indian Journal of pathology and microbiology. Letter to editor, Year 2011, volume 54 Issue 1 , Page 230 -232 


\section{CASE REPORT}

\section{Gross: Lumpectomy Specimen showing cystic areas}

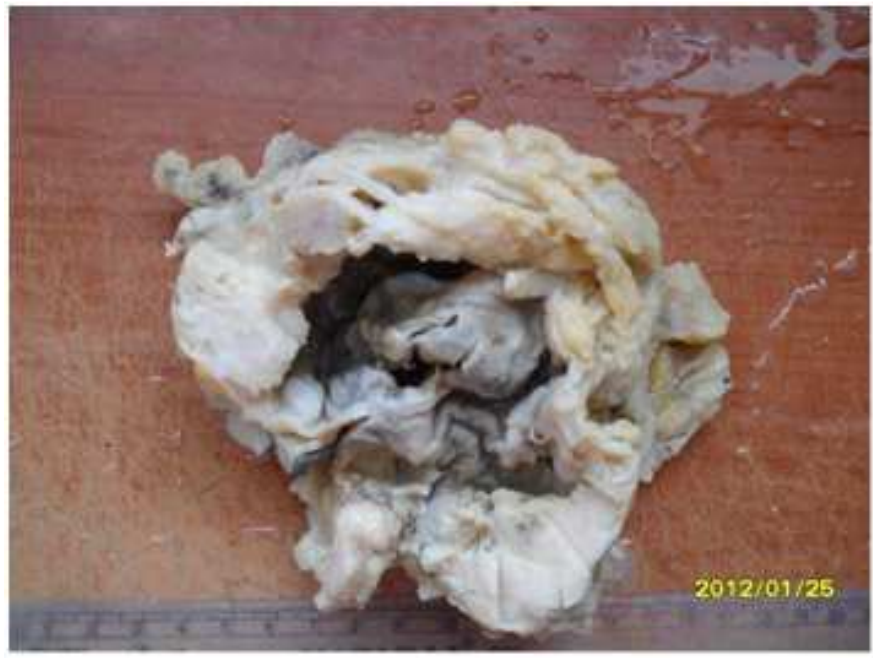

High Power View

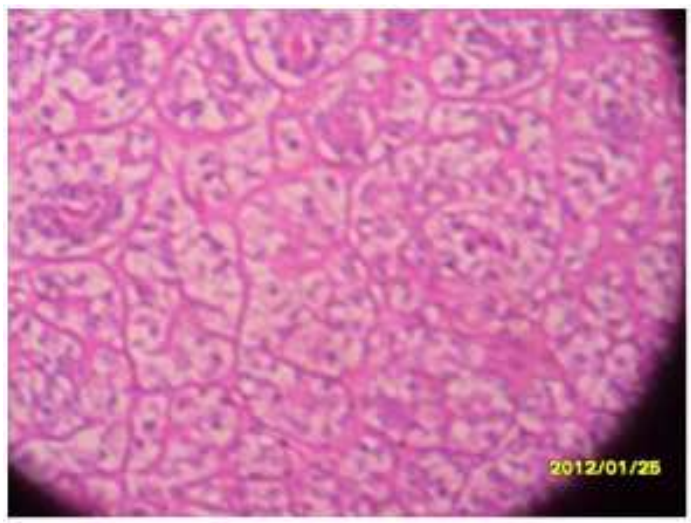

Low Power view

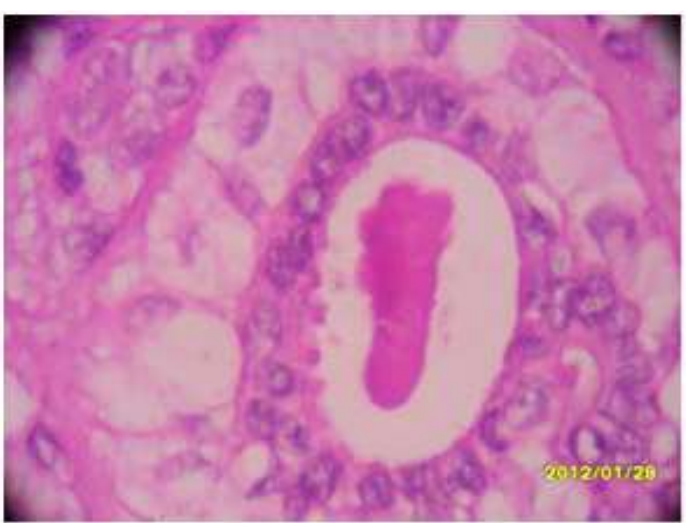

